\title{
Gastrointestinal stromal tumours at Inkosi Albert Luthuli Central Hospital from 2005 to 2015
}

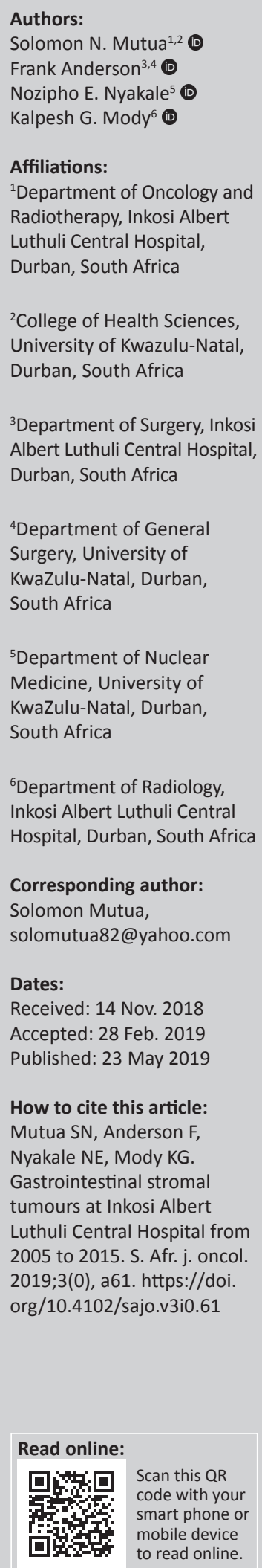

Background: Gastrointestinal stromal tumour (GIST) is the commonest mesenchymal malignancy of the gastrointestinal tract. Patient demographics and outcomes following imatinib therapy in South Africa are unknown.

Aim: To establish the patient demographics of GIST and the clinical outcomes following imatinib therapy.

Setting: Inkosi Albert Luthuli Central Hospital (IALCH), Durban, South Africa.

Methods: A quantitative, retrospective, descriptive chart review study was conducted. The study population included patients with a histologic diagnosis of GIST who presented between January 2005 and December 2015 to the facility. Only patients who received imatinib were included in the clinical outcome analysis.

Results: Sixty-nine patients were seen during the study period. The mean (SD) age at diagnosis was 57.3 (13.5) years. The male gender (53.6\%) was predominant, the black ethnic group (53.6\%) was the commonest and the stomach $(69.6 \%)$ was the most common disease site. Localised disease $(53.6 \%)$ was the commonest disease category, while high risk $(29.7 \%)$ and intermediate risk $(29.7 \%)$ were the majority risk categories. Thirty-six $(52.2 \%)$ patients received imatinib with a median (IQR) follow-up time of 20.5 (38) months. Eighty-one per cent of patients with localised disease remained in remission after adjuvant imatinib, and $18.2 \%$ developed metastatic recurrence. Among patients with locally advanced disease, $81.8 \%$ attained partial response on neoadjuvant imatinib, while $9.1 \%$ had stable disease. Most (75\%) patients with metastatic disease attained partial response as the best response to imatinib. The most common adverse effects were anaemia and fluid overload.

Conclusion: At IALCH, GIST is more common in the male gender, black ethnic group and in the stomach. The majority of localised and locally advanced GIST patients have favourable outcomes on imatinib. However, most metastatic GIST patients eventually develop resistance to imatinib necessitating further treatment options.

Keywords: imatinib therapy; gastrointestinal stromal tumours; imatinib; sunitinib; Inkosi Albert Luthuli Central Hospital.

\section{Introduction}

Gastrointestinal stromal tumour (GIST) is a malignancy that arises from the mesenchyme of the gastrointestinal tract (GIT). Despite being the commonest mesenchymal malignancy in the GIT, it constitutes less than $1 \%$ of all GIT malignancies. ${ }^{1,2,3,4}$ Globally, the epidemiology of GIST is not well known. According to a population-based study from the United States, the age at diagnosis ranges from 20 to 98 years, with a mean age of 62.9 years. Eighty per cent of patients are diagnosed above the age of 50, with those presenting below the age of 20 being rare. This study has also shown a slight male predominance in the prevalence of GIST with $54 \%$ of the cases occurring in male patients. Seventy-four per cent of patients were white people, $14 \%$ black people and $15 \%$ belonged to other ethnic groups. ${ }^{5}$ The interstitial cells of Cajal, found in the myenteric plexus of the GIT, are the cells of origin of this malignancy. ${ }^{6,7}$ Gastrointestinal stromal tumour occurs throughout the GIT with $60 \%$ to $70 \%$ occurring in the stomach and $30 \%$ in the small intestine ${ }^{1,2}$ Of these tumours, $5 \%$ have been found to arise from the colon, $4 \%$ from the rectum and $1 \%$ from the oesophagus. ${ }^{5}$ There is a lack of information on the demographic and anatomic distribution of GIST in South Africa.

Several genetic mutations are responsible for the pathogenesis of GIST. c-KIT and platelet-derived growth factor receptor alpha (PDGFRA) mutations are the commonest, occurring in $80 \%$ and

Copyright: @ 2019 . The Authors. Licensee: AOSIS. This work is licensed under the Creative Commons Attribution License. 
$5 \%-10 \%$ of GISTs, respectively. ${ }^{8,9,10}$ These mutations are mutually exclusive $\mathrm{e}^{10} ; 10 \%-15 \%$ of GISTs lack both $c$-KIT and PDGFRA mutations and are known as wild-type GIST. ${ }^{11}$ These constitute mutations on succinate dehydrogenase $(S D H)$, $B R A F$ and neurofibromatosis type 1 (NF-1) genes. ${ }^{12,13,1,4,15,16,17,18}$ A South African series found $c-K I T$ and PDGFRA mutations in $78.3 \%$ of GISTs, while wild-type GIST was found in $21.7 \%$ of the population. ${ }^{19}$ Immunohistochemical staining for CD117 (KIT immunostaining), DOG1 and CD34, and genetic molecular testing for $c-K I T$ and/or PDGFRA mutations are used to diagnose GIST. ${ }^{4,8,20}$ Both CD34 and DOG1 are highly expressed in GIST, and DOG1 immunostaining may be useful for cases that cannot be categorised as GIST based on CD117 immunostaining. ${ }^{8,20}$

Despite being the treatment modality of choice, surgery alone is not curative for all GIST patients. Half of the patients with operable disease undergoing surgery alone develop local or metastatic recurrence. ${ }^{21}$ The 5 -year survival rate of these patients is approximately $50 \%,{ }^{22}$ while the median time to recurrence after resection alone of high-risk GIST is 2 years. ${ }^{8,23}$ These facts underscore the importance of additional therapy in the management of this disease. Imatinib, a small molecule tyrosine kinase inhibitor (TKI) with activity against $c-K I T$ and PDGFRA tyrosine kinases, is the first-line treatment agent for GIST patients with locally advanced and metastatic disease. Imatinib use has improved the overall survival of these patients. ${ }^{6}$

Several prognostic factors have been shown to affect the outcome of patients treated with surgery. These include tumour size, mitotic index, anatomic disease location and tumour rupture (before or during surgery). ${ }^{1,29,24,25,26}$ Tumour size and mitotic index are used to stratify GISTs, according to their postoperative risk of recurrence and metastasis, into very low, low, intermediate and high risk. ${ }^{9}$ High-risk GIST patients who receive adjuvant imatinib for 3 years have 5 -year overall and recurrence free survival rates of $92 \%$ and $65.6 \%$, respectively, ${ }^{27}$ while those with metastatic GIST on palliative imatinib have a 9 -year survival rate of $35 \%-49 \%{ }^{28}$ Locally advanced GISTs are often unresectable or borderline resectable with significant surgical morbidity. Cytoreduction with neoadjuvant imatinib reduces this surgical morbidity. ${ }^{29}$ It is generally accepted that patients who require neoadjuvant imatinib should receive imatinib postoperatively for a cumulative period of 3 years. After prolonged exposure to imatinib, patients with metastatic GIST eventually develop resistance to it. Sunitinib and regorafenib are small molecule TKIs approved to treat patients with advanced GIST in the second- and third-line settings, respectively. ${ }^{8,30,31}$ Sorafenib, nilotinib, dasatinib and pazopanib are TKIs that have shown activity in phase II trials. ${ }^{32,33,34,35}$ There is a lack of information on the clinical outcomes and prognostic factors of GIST patients treated with imatinib in South Africa.

\section{Aim}

To establish the patient demographics of GIST and the clinical outcomes following imatinib therapy.

\section{Methodology}

This was a quantitative, retrospective, descriptive chart review study that was conducted at Inkosi Albert Luthuli Central Hospital (IALCH). The study population included patients with a histologic diagnosis of GIST who presented between January 2005 and December 2015 to the facility. Only patients who received imatinib were included in the clinical outcome analysis. This analysis was performed separately for patients with localised, locally advanced and metastatic disease. Localised disease was defined as operable disease with no distant spread. Locally advanced disease was defined as inoperable or borderline operable disease with no distant spread, and metastatic disease was defined as disease with distant spread at the time of diagnosis. There was no direct patient contact in this study, and therefore, informed consent was not required.

Patient data were collected from an electronic medical records database (speed minor) using ICD-10 codes. Age, gender, ethnicity, anatomic location, immunohistochemical stain, disease category, risk category (for localised disease), clinical outcome, adverse effects and death were analysed. Patients with localised disease were stratified according to their postoperative risk of recurrence and metastasis, using the American National Institutes of Health criteria. ${ }^{9}$

Clinical outcome analysis was based on the evaluation of serial computer tomography (CT) and positron emission tomography (PET) scan imaging. Patients with localised disease were analysed as either being in remission, local recurrence or metastatic recurrence. Objective responses (partial response, stable disease and progressive disease) for locally advanced and metastatic disease were analysed using Response Evaluation Criteria in Solid Tumors (RECIST 1.1) ${ }^{36}$ and European Organisation for Research and Treatment of Cancer (EORTC) $)^{37}$ criteria. Factors affecting clinical outcomes were analysed for each disease category. Adverse effects experienced by more than $5 \%$ of patients on imatinib were analysed. Stratification of adverse effects was done for the two most common adverse effects: anaemia according to the Common Terminology Criteria of Adverse Effects (CTCAE) ${ }^{38}$ and fluid overload according to the site of fluid overload. Patients were censored at the time of loss to follow-up, when they got transferred to a different hospital, placed on best supportive care only and at the end of the study period.

\section{Statistical analysis and ethics}

IBM SPSS Statistics version 25 was used to perform statistical analysis. Descriptive statistics (frequencies and percentages) were calculated to summarise categorical data. Measures of central tendency (mean and median) and measures of dispersion (standard deviation and interquartile range) were calculated for numerical variables. A histogram and bar graphs were used to display distribution of numerical data. One-sample Kolmogorov-Smirnov test was used to test the distribution of age. Spearman's rho correlation coefficient 
was calculated to measure the strength of linear correlation between age and number of adverse effects. Kruskal-Wallis test was used to test the null hypothesis that number of adverse effects is the same on average for patients in different disease categories and risk categories. Mann-Whitney U test was used to test the null hypothesis that the number of adverse effects is the same for male and female patients. Fisher's exact test was used to test for association between categorical variables. A $p$-value less than 0.05 was considered statistically significant.

\section{Ethical considerations}

Ethical approval was granted by the KwaZulu-Natal Department of Health (reference number: HRKM234/16 KZ_2016RP25_355) and the Biomedical Research Ethics Committee of the University of KwaZulu-Natal.

\section{Results}

Between January 2005 and December 2015, 69 patients with GIST were evaluated at IALCH. The mean (SD) age at diagnosis (Figure 1) was 57.3 (13.5) years. Seventy-five per cent of the patients were above the age of 50 , and only one patient was younger than 20 years at the time of diagnosis. In the study population (Table 1), there was a slight male predominance $(53.6 \%)$, and majority of the patients were of black ethnicity (53.6\%). The stomach (69.6\%) was the commonest disease site followed by the small intestine (13\%). The urinary bladder (1.4\%) and oesophagus $(1.4 \%)$ were the least common anatomic sites of disease. Out of the three disease categories (Table 1), localised disease (53.6\%) was the commonest, while high risk (29.7\%) and intermediate risk $(29.7 \%)$ were the commonest observed postoperative risk categories. CD117 (Figure 2) was the

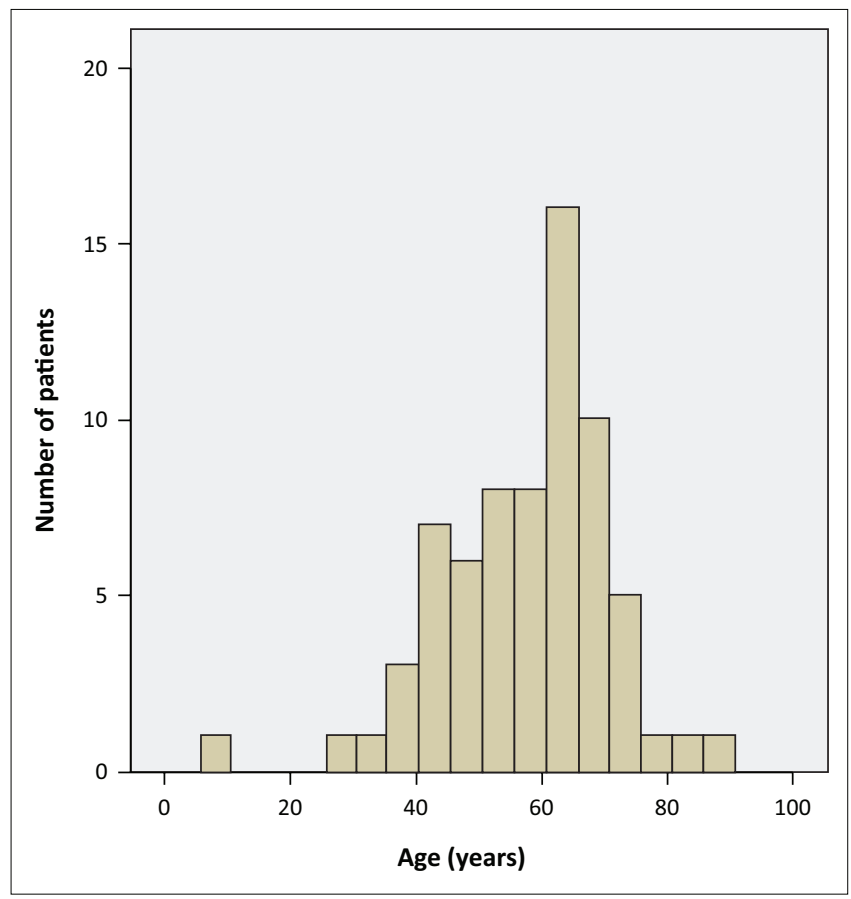

Mean $=57.26$; standard deviation $=13.519 ;$ total number of patients $=69$. FIGURE 1: Age distribution. commonest observed immunohistochemical marker in this patient population, with $21.7 \%$ of the cases having unknown immunohistochemical expression.

Thirty-six (52.2\%) patients were treated with imatinib during the study period (Figure 3). The follow-up time ranged from 1 month to 108 months with a median (IQR) of 20.5 (38) months. Eleven patients were lost to follow-up during the study period. The median (IQR) duration of imatinib treatment was 12 (10) months, 18.5 (36.1) months and 43.5 (35) months for localised, locally advanced and metastatic disease, respectively. Eleven patients with localised disease received imatinib in the adjuvant setting. Six of these had

TABLE 1: Patient demographics and disease characteristics.

\begin{tabular}{lc}
\hline Variable & Number (\%) \\
\hline Gender & $37(53.6)$ \\
Male & $32(46.4)$ \\
Female & \\
Ethnicity & $37(53.6)$ \\
Black people & $17(24.6)$ \\
Asian people & $9(13.0)$ \\
White people & $5(7.2)$ \\
Mixed race people & $1(1.4)$ \\
Unknown & \\
Anatomic location & $48(69.6)$ \\
Stomach & $9(13.0)$ \\
Small intestine & $4(5.8)$ \\
Mesentery & $2(2.9)$ \\
Rectum & $2(2.9)$ \\
Peritoneum & $1(1.4)$ \\
Oesophagus & $1(1.4)$ \\
Urinary bladder & $2(2.9)$ \\
Unknown & \\
Disease category & $18(53.6)$ \\
Localised & $13(18.8)$ \\
Locally advanced & $18(26.1)$ \\
Metastatic & $1(1.4)$ \\
Unknown & \\
Risk category (localised disease) & $2(5.4)$ \\
Lery low risk & $11(29.7)$ \\
Hntermediate risk &
\end{tabular}

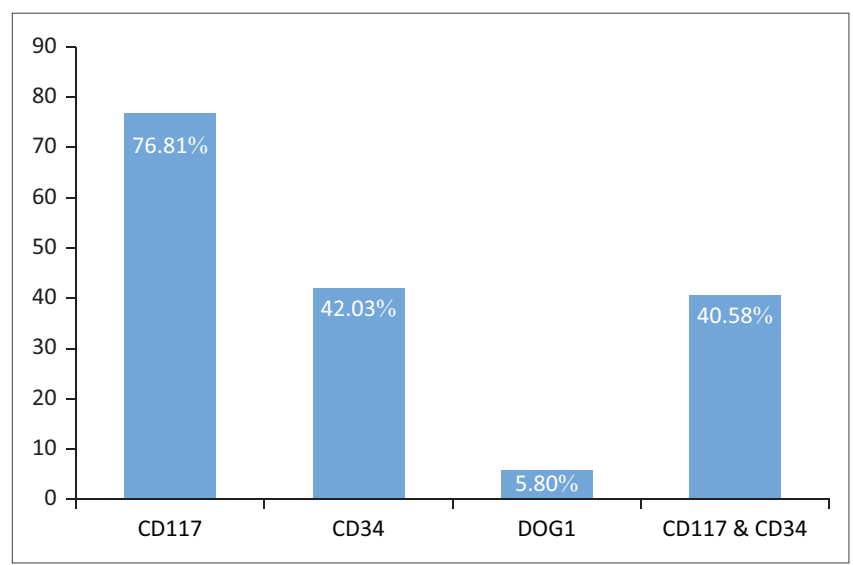

FIGURE 2: The immunohistochemical expression of gastrointestinal stromal tumour. 


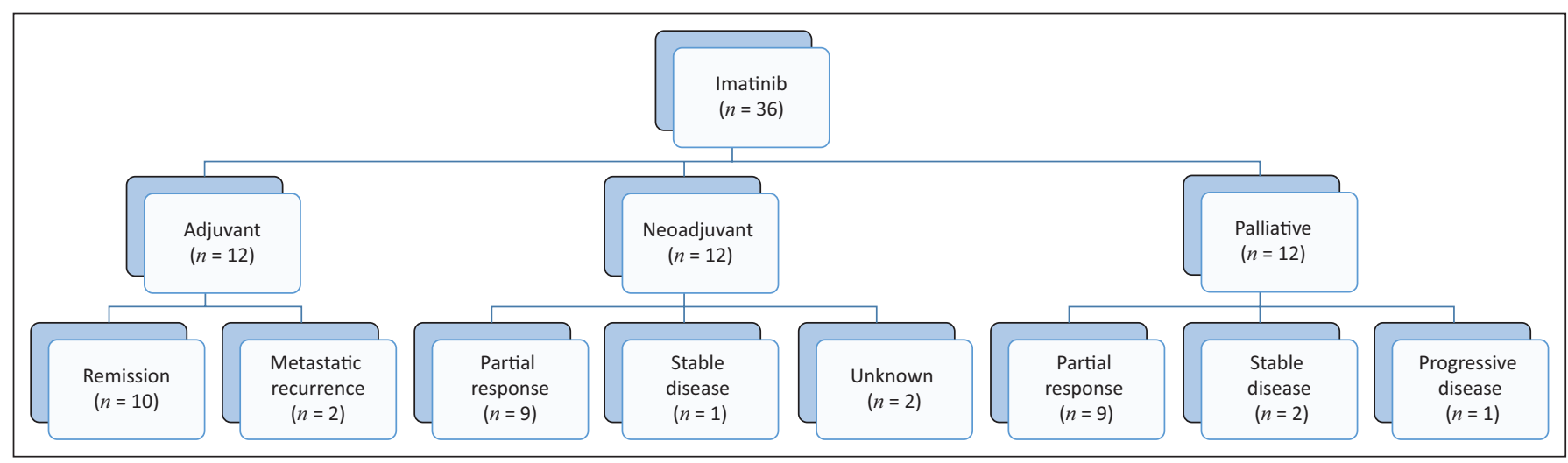

FIGURE 3: Imatinib indications and objective responses.

high-risk disease, while five had intermediate-risk disease. Five patients with high-risk disease did not receive imatinib. One of them died of postoperative complications, while the remaining four were not referred for adjuvant therapy. Nine $(81.8 \%)$ of the 11 patients treated with adjuvant imatinib remained in remission, and two (18.2\%) developed metastatic recurrence. Seven $(77.8 \%)$ of the patients who remained in remission had the stomach as the primary disease site, five (55.6\%) had intermediate risk disease and six $(66.7 \%)$ had complete gross resection. Three patients in remission had incomplete surgical resection (two with microscopic and one with macroscopic residual disease). Both patients with metastatic recurrence had high-risk disease. One of them declined adjuvant imatinib after receiving it for 12 months. There were no locoregional recurrences observed among these patients.

All 13 patients with locally advanced disease were treated with imatinib. One of these $(9.1 \%)$ had primary resection and remained in remission after adjuvant imatinib. The remaining 12 patients received neoadjuvant imatinib for a median (IQR) of 8.5 (11.25) months (range 2-36 months). Partial response (PR) was observed in nine (81.8\%) patients. Six of these underwent surgical resection, two declined surgery and one was medically inoperable. Most patients (four) who attained partial response had disease located in the stomach. Stable disease (SD) was observed in one (9.1\%) patient who subsequently underwent debulking surgery. None of the patients with locally advanced GIST had complete response (CR) or progressive disease (PD) after neoadjuvant imatinib. The outcome of two patients with locally advanced disease was not known.

A total of 12 (66.7\%) patients with metastatic disease received imatinib treatment, while six did not. Two of these six patients were in a poor clinical condition to receive imatinib. It is unknown why the remaining four patients did not receive imatinib. Out of the patients that received imatinib, nine (75\%) attained PR, two (16.7\%) attained SD and one $(8.3 \%)$ developed PD. Eight $(88.9 \%)$ of the patients that attained PR, and two patients that attained SD had the primary disease in the stomach. The patient that developed PD had the peritoneum as the primary site of disease. Nine (75\%) patients with metastatic disease eventually progressed on imatinib with a median (IQR) time to progression of 40.5 (47.25) months (range 3.5-84 months). Seven of these patients were placed on an increased dose imatinib (800 mg daily), while the remaining two were placed on best supportive care because of their poor clinical condition. Four patients developed disease progression on increased dose imatinib and three patients could not tolerate it. Across all disease categories, there was a total of three deaths recorded during the study period. Two of these deaths occurred as a result of surgical complications and one as a result of disease progression.

Out of the 36 patients treated with imatinib, 31 (86.1\%) experienced adverse effects. The two commonest adverse effects (Figure 4) were anaemia (83\%) and fluid overload (31\%). Grades III, II and I anaemia were found in $43.3 \%$, $23.3 \%$ and $33.3 \%$ of the patients, respectively. Grades IV and $\mathrm{V}$ anaemia were not observed in this patient population. Fluid overload was in the form of pedal oedema $(72.7 \%)$, facial oedema $(18.2 \%)$, ascites $(18.2 \%)$, hand oedema $(9.1 \%)$ and pleural effusion (9.1\%). Male patients $(64.5 \%)$ had more adverse effects than their female counterparts. Patients with metastatic disease experienced the most adverse effects with a median of 4 (range $0-10$ ) adverse effects. These were followed by those with locally advanced and localised disease with a median of 2 (range 0-4) and 1 (range 0-6) adverse effects, respectively. Risk category and age, however, did not influence the occurrence of adverse effects in this patient population. A total of five $(13.9 \%)$ patients had their imatinib treatment interrupted because of adverse effects. One of these was as a result of Grade III anaemia, two as a result of fluid overload (ascites and pleural effusion) and two as a result of Grade III diarrhoea.

\section{Discussion}

This study describes the demographics of GIST patients and their treatment outcomes after imatinib therapy at IALCH. Similar to previous research, ${ }^{5,39}$ GIST has been found to occur mainly in older patients with three quarters of the study population having been diagnosed above the age of 50. This study has also established that GIST is rarely diagnosed below the age of 20 with only $1.4 \%$ of the study population 


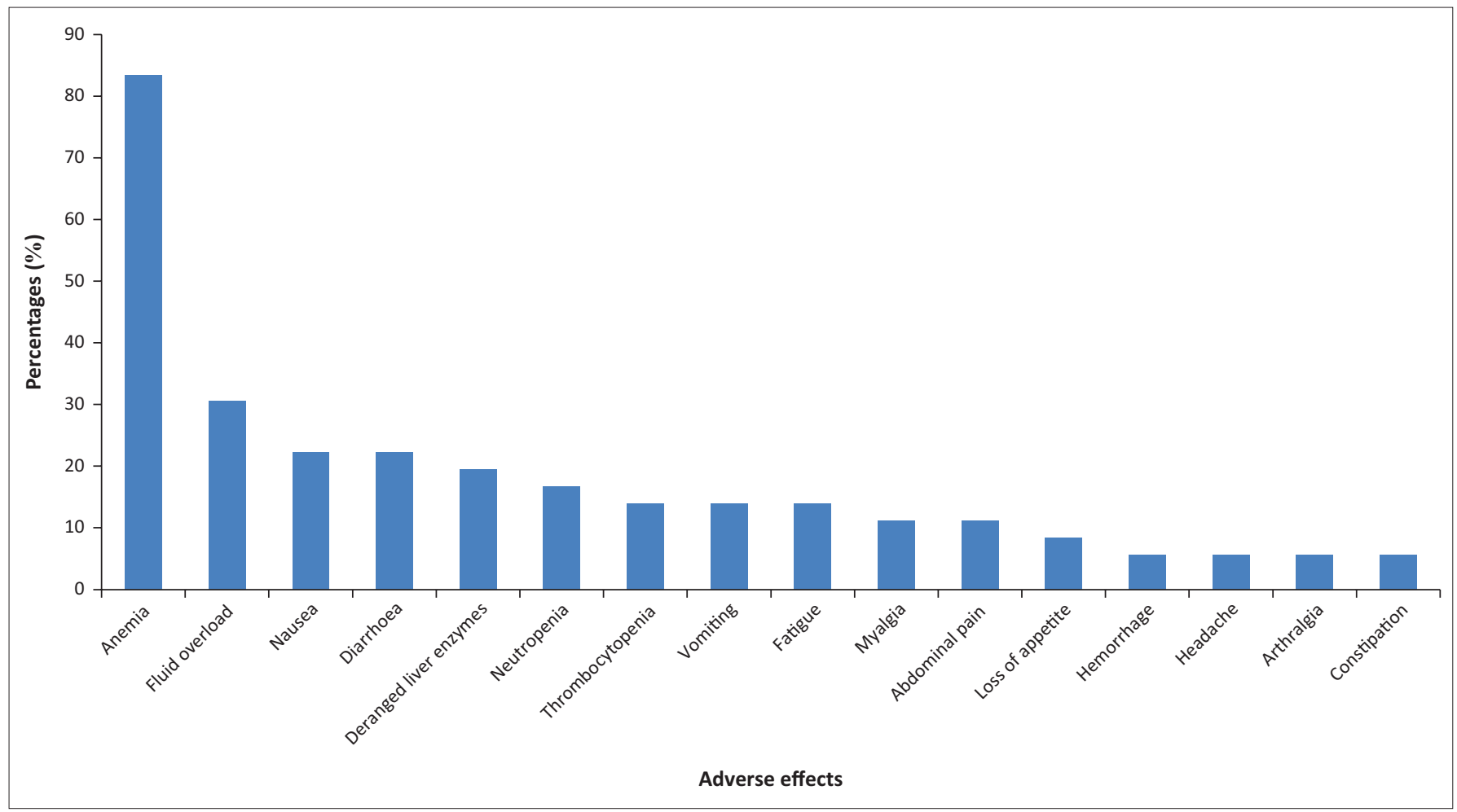

FIGURE 4: Adverse effects occurring in more than $5 \%$ of patients on imatinib.

diagnosed below this age. The first ever population-based study using the Surveillance Epidemiology and End Result (SEER) data showed a slight male predominance with $54 \%$ of the cases occurring in male and $46 \%$ in female patients. ${ }^{5}$ Contrary to this finding, there was a significant male predominance in a Pretoria series with a male-to-female ratio of 1.5:1. ${ }^{39}$ In our local setting, this study has found a slight male predominance, commensurate with the SEER study. The ethnic distribution of GIST in this study is proportional to the local population demographics, a finding similar to previous research. ${ }^{5,39}$

In this study cohort, the stomach was the commonest anatomic site affected by GIST followed by the small intestine, similar to previous research. However, the small intestine was affected at a much lower percentage in our local setting and the Pretoria series, compared to previous research that found the small intestine affected in $30 \%$ of patients. ${ }^{1,39}$ The oesophagus, rectum, mesentery, peritoneum and urinary bladder were the other anatomic sites involved by GIST in the local setting. Anatomic sites described in literature that were not affected by GIST in the local setting include the colon, anus, appendix, omentum and gall bladder. ${ }^{5}$ Among patients with localised disease, high and intermediate risks were the majority postoperative risk categories. Patients with high-risk disease are the group with the highest risk of recurrence and metastasis after surgery and would therefore benefit from adjuvant imatinib therapy. ${ }^{24,27}$ This finding underscores the role of imatinib in the local setting.

CD117 (KIT) was the commonest expressed immunohistochemical marker in our study. However, CD117 was expressed at a lower percentage compared to previous research where it was expressed in more than $90 \%$ of the population. ${ }^{1,2}$ This can be explained by the fact that a significant proportion of GIST cases in our setting had unknown immunohistochemical expression. CD34 and DOG1 were also expressed in a minority of GIST cases, contrary to previous research. ${ }^{20,40}$ At IALCH, DOG1 staining is only done as a confirmatory test, when CD117 and CD34 staining are equivocal. This explains the low expression observed in our study. DOG1 staining was done for four GIST cases, all of which stained positive. The occurrence of c-KIT, PDGFRA, SDH, BRAF and NF1 mutations could not be established because genetic molecular mutation testing is not routinely done at IALCH.

At IALCH, adjuvant imatinib is prescribed for 3 years to localised GIST patients with postoperative high-risk and select intermediate-risk disease. Adjuvant imatinib for a duration of 3 years reduces the risk of local and metastatic recurrence in high-risk GIST patients. ${ }^{27}$ Incidentally, patients with localised disease that required adjuvant imatinib presented during the latter part of the study. This explains the short duration of adjuvant imatinib therapy because patients were censored at the end of the study period. Throughout the study period, most patients with localised disease remained in remission after adjuvant imatinib. The majority of patients in remission had disease located in the stomach. It is, however, not clear whether the stomach anatomic site contributed to this positive outcome because other anatomic disease sites were not sufficiently represented for comparison. Three patients with localised GIST attained remission after imatinib therapy despite having residual disease postoperatively. In this era of imatinib, the optimal management of such patients with 
positive margins is still unclear. ${ }^{41}$ All patients that developed metastatic recurrence had high-risk disease, in keeping with previous studies that established tumour size and mitotic index as strong risk factors for treatment failure. ${ }^{1,2}$ One of the patients that developed metastatic recurrence did not complete adjuvant treatment. This patient may have benefited from completing adjuvant imatinib. It is important, therefore, to educate patients on the benefits of imatinib therapy.

Imatinib treatment has enabled a number of GIST patients with locally advanced and metastatic disease to undergo surgical resection, thereby improving their clinical outcomes. Patients with locally advanced GIST who respond to neoadjuvant imatinib benefit from surgery. Surgery may also be considered in patients with limited PD on neoadjuvant imatinib. In contrast, surgery has little to offer to patients with generalised PD after receiving neoadjuvant imatinib..$^{8,42}$ In the local setting, most patients with locally advanced GIST required neoadjuvant imatinib, with the majority of them attaining PR. This is in keeping with previous research in which $60 \%$ of the patients attained PR after neoadjuvant imatinib. In the same study, SD (28\%) and PD (12\%) were also observed. ${ }^{43}$ Most of the patients that attained PR had disease located in the stomach. However, similar to the localised disease cohort, the prognostic significance of this anatomic location could not be established.

Metastatic GIST is often an incurable disease. The majority of patients with metastatic GIST in our cohort initially attained favourable responses on imatinib. This is commensurate with initial studies where imatinib has produced favourable objective responses in more than $50 \%$ of patients with advanced GIST. ${ }^{6,44,45,46}$ To achieve response induction, imatinib is safe and efficacious at an initial standard dose of $400 \mathrm{mg}$ daily. ${ }^{44,46}$ Dose escalation to $800 \mathrm{mg}$ daily is indicated for patients progressing on $400 \mathrm{mg} .{ }^{45}$ Almost all patients with metastatic GIST in our study progressed on the standard dose imatinib leading to dose escalation. However, these patients either progressed on or could not tolerate high-dose imatinib.

Most patients treated for metastatic disease eventually develop resistance to imatinib leading to disease progression and death. Sunitinib has been shown to improve outcomes in patients that have progressed on imatinib. In this second-line setting, the median time to tumour progression was significantly improved with sunitinib (27.3 weeks) compared to placebo (6.4 weeks). ${ }^{30}$ At IALCH, sunitinib is not available for the treatment of patients with GIST and leaves patients with advanced GIST without systemic therapeutic options once they develop resistance to imatinib. This situation underscores the need to avail sunitinib as the second-line treatment of GIST patients in the public health sector.

The majority of patients treated with imatinib in this study experienced adverse effects. However, grades of adverse effects were not documented for all patients that experienced them. Imatinib is well tolerated with most adverse effects being mild to moderate, seldom requiring treatment interruption or dose adjustment. Serious adverse effects like bone marrow suppression, impaired liver function tests and neutropenic fever have been found to be rare with this molecule. ${ }^{47}$ In the local setting, imatinib is also well tolerated with only five patients having their treatment interrupted because of adverse effects. However, anaemia, which is as a result of bone marrow suppression, was the commonest adverse effect observed in patients treated with imatinib in this study. Furthermore, the majority of these patients had Grade III anaemia. It is not clear why anaemia was common in this patient population. Male gender and metastatic disease category were shown to increase the occurrence of adverse effects in our local population. However, age and risk category did not influence the occurrence of adverse effects in our local patient population.

This study has some weaknesses. Firstly, its retrospective nature subjects it to potential bias. Secondly, incomplete record-keeping with regard to death, pathology reports and failure to treat several patients with imatinib hampered the study. While it is possible that more patients died during the study period, only three deaths were recorded because most deaths that occur outside IALCH are not captured in the hospital records. Seven patients with localised disease had unknown risk categories because of lack of pathology information vital in decision-making (tumour size and mitotic index). In addition, there was no documentation as to why eight patients (four with high-risk and four with metastatic GIST) were not treated with imatinib. We recommend that a mechanism be put in place that will enable peripheral hospitals or caregivers to inform IALCH of patients' demise. Open communication between the oncologist and pathologist is encouraged to ensure that vital information is provided for treatment decisionmaking. Clinicians are also encouraged to ensure adequate documentation of decisions made in the clinic. Thirdly, the patient population in this study was small. A larger, multiinstitution study is recommended in order to better describe the different variables of this rare disease.

\section{Conclusion}

This study describes the patient demographics and clinical outcomes after treatment with imatinib in GIST patients at IALCH. The majority of GIST patients were diagnosed above the age of 50, and male patients were slightly more numerous than their female counterparts. Most patients belonged to the black ethnic group, a fact attributable to the local population distribution. The stomach was the commonest anatomic site affected by GIST. High- and intermediate-risk GISTs were the commonest risk categories among patients with resected disease, with a significant number of patients having presented with locally advanced and metastatic disease. Therefore, imatinib therapy has an important role in the local setting. As expected, patients with advanced GIST (locally advanced and metastatic disease) initially attained favourable responses during imatinib therapy thereby improving their clinical outcomes. Imatinib was well 
tolerated with anaemia being the commonest adverse effect observed. Clinicians should, therefore, anticipate and manage anaemia timeously to improve patient tolerance to imatinib. The majority of patients with metastatic GIST eventually develop resistance to imatinib. It is therefore important to avail sunitinib, as the second-line treatment agent, in the public health sector.

\section{Acknowledgements}

The authors thank Fikile Nkwanyana (University of KwaZuluNatal) who provided statistical support for this study.

\section{Competing interests}

The authors declare that they have no financial or personal relationships that may have inappropriately influenced them in writing this article.

\section{Authors' contributions}

S.N.M. was the primary author responsible for the research concept, research design, study protocol, literature review, data collection, data analysis, initial draft of the manuscript, manuscript editing and final draft of the manuscript. F.A. was responsible for the research concept, data collection and proofreading of the initial draft and final draft of the manuscript. Both N.E.N. and K.G.M. assisted with proofreading the initial draft and final draft of the manuscript.

\section{Funding}

This research received no specific grant from any funding agency in the public, commercial or not-for-profit sectors.

\section{Disclaimer}

The views expressed in this article are the authors' own and not an official position of the institution.

\section{References}

1. Miettinen M, Makhlouf H, Sobin LH, Lasota J. Gastrointestinal stromal tumors of the jejunum and ileum: A clinicopathologic, immunohistochemical, and molecula genetic study of 906 cases before imatinib with long-term follow up. Am J Surg Pathol. 2006;30(4):477-489. https://doi.org/10.1097\%2F00000478-20060400000008

2. Miettinen M, Sobin LH, Lasota J. Gastrointestinal stromal tumors of the stomach: A clinicopathologic, immunohistochemical, and molecular genetic study of 1765 cases with long-term follow up. Am J Surg Pathol. 2005;29(1):52-68. https://doi. org/10.1097\%2F01.pas.0000146010.92933.de

3. Rammohan A, Sathyanesan J, Rajendran K, et al. A gist of gastrointestinal stromal tumors: A review. WJGO. 2013;5(6):102-112. https://doi.org/10.4251\%2Fwjgo. v5.i6.102

4. Demetri GD, Von Mehren M, Antonescu CR, et al. NCCN Task Force report: Update on the management of patients with gastrointestinal stromal tumors. J Natl Compr Canc Netw. 2010;8(Suppl 2):S1-S41. https://doi.org/10.6004\%2Fjnccn.2010.0116

5. Tran T, Davila JA, El-Serag HB. The epidemiology of malignant gastrointestinal stromal tumors: An analysis of 1,458 cases from 1992 to 2000. Am J Gastroenterol. 2005;100(1):162-168. https://doi.org/10.1111\%2Fj.1572-0241.2005.40709.x

6. Demetri GD, Von Mehren M, Blanke CD, et al. Efficacy and safety of imatinib mesylate in advanced gastrointestinal stromal tumors. N Engl J Med. 2002;347(7):472-480. https://doi.org/10.1056\%2Fnejmoa020461

7. Tzen $\mathrm{CY}$, Wang JH, Huang YJ, et al. Incidence of gastrointestinal stromal tumors: A retrospective study based on immunohistochemical and mutational analyses. Dig Dis Sci. 2007;52(3):792-797. https://doi.org/10.1007\%2Fs10620-006-9480-y

8. Von Mehren M, Randall RL, Benjamin RS, et al. Soft tissue sarcoma, version 2.2016, NCCN clinical practice guidelines in oncology. J Natl Compr Canc Netw. 2016;14(6):758-786. https://doi.org/10.6004\%2Fjnccn.2016.0078
9. Fletcher CD, Berman JJ, Coreless $C$, et al. Diagnosis of gastrointestinal stromal tumors: A consensus approach. Int J Surg Pathol. 2002;10(2):81-89. https://doi.org/ tumors: A consensus approach. Int J

10. Heinrich $\mathrm{MC}$, Corless $\mathrm{CL}$, Duensing $\mathrm{A}$, et al. PDGFRA activating mutations in gastrointestinal stromal tumors. Science. 2003;299(5607):708-710. https://doi. org/10.1126\%2Fscience.1079666

11. Corless $\mathrm{CL}$, Fletcher JA, Heinrich MC. Biology of gastrointestinal stromal tumors. J Clin Oncol. 2004;22(18):3813-3825. https://doi.org/10.1200\%2Fjco.2004. 05.140

12. Italiano A, Chen C-L, Sung Y-S, et al. SDHA loss of function mutations in a subset of young adult wild-type gastrointestinal stromal tumors. BMC Cancer. 2012;12(1):408 https://doi.org/10.1186\%2F1471-2407-12-408

13. Pantaleo MA, Astolfi A, Urbini M, et al. Analysis of all subunits, SDHA, SDHB, SDHC SDHD, of the succinate dehydrogenase complex in KIT/PDGFRA wild-type GIST. Eur J Hum Genet. 2014;22(1):32. https://doi.org/10.1038\%2Fejhg.2013.80

14. Boikos SA, Pappo AS, Killian J, et al. Molecular subtypes of kit/pdgfra wild-type gastrointestinal stromal tumors: A report from the national institutes of health gastrointestinal stromal tumor clinic. JAMA Oncol. 2016;2(7):922-928. https:// doi.org/10.1001\%2Fjamaoncol.2016.0256

15. Agaram NP, Wong GC, Guo T, et al. Novel V600E BRAF mutations in imatinib-naïve and imatinib-resistant gastrointestinal stromal tumors. Genes Chromosomes Cancer. 2008;47(10):853-859. https://doi.org/10.1002\%2Fgcc.20589

16. Agaimy A, Terracciano L, Dirnhofer $S$, et al. V600E BRAF mutations are alternative early molecular events in a subset of KIT/PDGFRA wild-type gastrointestinal stromal tumors. J Clin Pathol. 2009;62(7):613-616. https://doi.org/10.1136\% 2Fjcp.2009.064550

17. Kinoshita $\mathrm{K}$, Hirota $\mathrm{S}$, Isozaki $\mathrm{K}$, et al. Absence of c-kit gene mutations in gastrointestinal stromal tumors from neurofibromatosis type 1 patients. J Pathol. 2004;202(1):80-85. https://doi.org/10.1002\%2Fpath.1487

18. Miettinen M, Fetsch JF, Sobin LH, Lasota J. Gastrointestinal stromal tumors in patients with neurofibromatosis 1: A clinicopathologic and molecular genetic study patients with neurofibromatosis 1: A clinicopathologic and molecular genetic study
of 45 cases. Am J Surg Pathol. 2006;30(1):90-96. https://doi.org/10.1097\%2F01. pas.0000176433.81079.bd

19. Baker G, Babb C, Schnugh D, et al. Molecular characterisation of gastrointestina stromal tumors in a South African population. Oncol Lett. 2013:5(1):155-160. https://doi.org/10.3892\%2Fol.2012.1013

20. Miettinen M, Wang Z-F, Lasota J. DOG1 antibody in the differential diagnosis of gastrointestinal stromal tumors: A study of 1840 cases. Am J Surg Pathol. 2009;33(9):1401-1408. https://doi.org/10.1097\%2Fpas.0b013e3181a90e1a

21. Eisenberg BL, Judson I. Surgery and imatinib in the management of GIST: Emerging approaches to adjuvant and neoadjuvant therapy. Ann Surg Oncol. 2004;11(5): 465-475. https://doi.org/10.1245\%2Faso.2004.09.011

22. DeMatteo RP, Lewis JJ, Leung D, Mudan SS, Woodruff JM, Brennan MF Two hundred gastrointestinal stromal tumors: Recurrence patterns and prognostic factors for survival. Ann Surg. 2000;231(1):51. https://doi.org/10.1097 \%2F00000658-200001000-00008

23. DeMatteo RP, Ballman KV, Antonescu CR, et al. Adjuvant imatinib mesylate after resection of localised, primary gastrointestinal stromal tumors: A randomised, double-blind, placebo-controlled trial. Lancet. 2009;373(9669):1097-1104. https://doi.org/10.1016/s0140-6736(09)60500-6

24. Rutkowski P, Nowecki ZI, Michej W, et al. Risk criteria and prognostic factors for predicting recurrences after resection of primary gastrointestinal stromal tumor. Ann Surg Oncol. 2007;14(7):2018-2027. https://doi.org/10.1245\%2Fs10434-0079377-9

25. Miettinen M, Lasota J, editors. Gastrointestinal stromal tumors: Pathology and prognosis at different sites. Semin Diagn Pathol. 2006;23(2):70-83. https://doi. org/10.1053\%2Fj.semdp.2006.09.001

26. Joensuu $\mathrm{H}$, Hohenberger $\mathrm{P}$, Corless $\mathrm{CL}$. Gastrointestinal stromal tumor. Lancet. 2013;382(9896):973-983. https://doi.org/10.1016\%2Fs0140-6736\%2813\%2 960106-3

27. Joensuu $H$, Eriksson $M$, Hall KS, et al. One vs three years of adjuvant imatinib for operable gastrointestinal stromal tumors: A randomized trial. JAMA. 2012;307(12): 1265-1272. https://doi.org/10.1001\%2Fjama.2012.347

28. Von Mehren M, Heinrich M, Joensuu H, Blanke C, Wehrle E, Demetri G. Follow-up results after 9 years (yrs) of the ongoing, phase II B2222 trial of imatinib mesylate (IM) in patients (pts) with metastatic or unresectable KIT+ gastrointestinal stromal tumors (GIST). J Clin Oncol. 2011;29(15_Suppl):10016. https://doi.org/10.1200\% 2Fjco.2011.29.15_suppl.10016

29. Fiore $M$, Palassini $E$, Fumagalli $E$, et al. Preoperative imatinib mesylate for unresectable or locally advanced primary gastrointestinal stromal tumors (GIST). Eur J Surg Oncol (ELSO). 2009;35(7):739-745. https://doi.org/10.1016\%2Fj.ejso. 2008.11.005

30. Demetri GD, Van Oosterom AT, Garett CR, et al. Efficacy and safety of sunitinib in patients with advanced gastrointestinal stromal tumor after failure of imatinib: $A$ randomised controlled trial. Lancet. 2006;368(9544):1329-1338. https://doi.org/ $10.1016 \% 2$ Fs0140-6736\%2806\%2969446-4

31. Demetri GD, Reichardt $P$, Kang $Y-K$, et al. Efficacy and safety of regorafenib for advanced gastrointestinal stromal tumors after failure of imatinib and sunitinib (GRID): An international, multicentre, randomised, placebo-controlled, phase 3 (GRID): An international, multicentre, randomised, placebo-controlled, phase 3
trial. Lancet. 2013;381(9863):295-302. https://doi.org/10.1016\%2Fs0140-6736\% trial. Lancet. 2013;

32. Park S, Ryu M, Ryoo B, et al. Sorafenib in patients with metastatic gastrointestinal tumors who failed two or more prior tyrosine kinase inhibitors: A phase II study of Korean gastrointestinal stromal tumors study group. Invest New Drugs. 2012;30(6):2377-2383. https://doi.org/10.1007\%2Fs10637-012-9795-9 
33. Montemurro $M$, Schoffski $P$, Reichardt $P$, et al. Nilotinib in the treatment of advanced gastrointestinal stromal tumors resistant to both imatinib and sunitinib. Eur J Cancer. 2009;45(13):2293-2297. https://doi.org/10.1016\%2Fj.ejca.2009. 04.030

34. Trent J, Wathen K, Von Mehren M, et al. A phase II study of dasatinib for patients with imatinib-resistant gastrointestinal stromal tumor (GIST). J Clin Oncol. 2011;29(15_Suppl):10006. https://doi.org/10.1200\%2Fjco.2011.29.15 suppl. 10006

35. Ganjoo KN, Villalobos V, Kamaya A, et al. A multicentre phase II study of pazopanib in patients with advanced gastrointestinal stromal tumors (GIST) following failure of at least imatinib and sunitinib. Ann Oncol. 2014;25(1):236-240. https://doi. org/10.1093\%2Fannonc $\% 2 \mathrm{Fmdt} 484$

36. Eisenhauer EA, Therasse $P$, Bogaerts J, et al. New response evaluation criteria in solid tumors: Revised RECIST guideline (version 1.1). Eur J Cancer. 2009;45(2): 228-247. https://doi.org/10.1016\%2Fj.ejca.2008.10.026

37. Young $H, B a u m R$, Cremerius $U$, et al. Measurement of clinical and subclinical tumor response using [18F]-fluorodeoxyglucose and positron emission tomography: Review and 1999 EORTC recommendations. Eur J Cancer. 1999:35(13):1773-1782. https://doi.org/10.1016\%2Fs0959-8049\%2899\%2900229-4

38. US Department of Health and Human Services, National Institutes of Health, National Cancer Institute. Common Terminology Criteria for Adverse Events (CTCAE) Version 4.0. European Organisation for Research and Treatment (CTCAE) Version 4.0. European Organisation for Researc ctc/CTCAE_4.03_2010-06-14_QuickReference_5x7.pdf.

39. Hartley R, Becker J, Van der Walt H, Luvhengo T. Gastro-intestinal stromal tumors (GISTs) - The Pretoria experience and a literature review. S Afr J Surg. 2011;49(3): 128-131.
40. Miettinen M, Lasota J. Gastrointestinal stromal tumors: Review on morphology, molecular pathology, prognosis, and differential diagnosis. Arch Pathol Lab Med. 2006;130(10):1466-1478.

41. Heinrich MC, Coreless CL. Gastric GI stromal tumors (GISTs): The role of surgery in the era of targeted therapy. J Surg Oncol. 2005;90(3):195-207. https://doi. org/10.1002\%2Fjso.20230

42. Raut CP, Posner M, Desai J, et al. Surgical management of advanced gastrointestinal stromal tumors after treatment with targeted systemic therapy using kinase inhibitors. J Clin Oncol. 2006;24(15):2325-2331. https://doi.org/10.1200\%2Fjco. 2005.05.3439

43. Blesius A, Cassier PA, Bertucci F, et al. Neoadjuvant imatinib in patients with locally advanced non metastatic GIST in the prospective BFR14 trial. BMC Cancer. 2011;11(1):72. https://doi.org/10.1186\%2F1471-2407-11-72

44. Verweij J, Casali PG, Zalcberg J, et al. Progression-free survival in gastrointestinal stromal tumors with high-dose imatinib: Randomised trial. Lancet. 2004;364(9440) 1127-1134. https://doi.org/10.1016\%2Fs0140-6736\%2804\%2917098-0

45. Zalcberg JR, Verweij J, Casali PG, et al. Outcome of patients with advanced gastrointestinal stromal tumors crossing over to a daily imatinib dose of $800 \mathrm{mg}$ after progression on $400 \mathrm{mg}$. Eur J Cancer. 2005;41(12):1751-1757. https://doi.org/ 10.1016\%2Fj.ejca.2005.04.034

46. Blanke CD, Rankin C, Demetri GD, et al. Phase III randomised, intergroup tria assessing imatinib mesylate at two dose levels in patients with unresectable or metastatic gastrointestinal stromal tumors expressing the kit receptor tyrosine kinase: S0033. J Clin Oncol. 2008;26(4):626-632. https://doi.org/10.1200\%2Fjco. 2007.13.4452

47. Guilhot F. Indications for imatinib mesylate therapy and clinical management Oncologist. 2004;9(3):271-281. https://doi.org/10.1634\%2Ftheoncologist.9-3-271 